\title{
Comparative chromosome maps between the stone curlew and three ciconiiform species (the grey heron, little egret and crested ibis)
}

Jinhuan Wang ${ }^{1}$, Weiting Su${ }^{1}$, Yi Hu' ${ }^{1}$, Shengbin Li ${ }^{2}$ Patricia C. M. O'Brien ${ }^{3}$, Malcolm A. Ferguson-Smith ${ }^{3}$, Fengtang Yang ${ }^{4^{*}}$ and Wenhui $\mathrm{Nie}^{1^{*}}$

\begin{abstract}
Background: Previous cytogenetic studies show that the karyotypes of species in Ciconiiformes vary considerably, from $2 n=52$ to 78 . Their karyotypes include different numbers of small to minute bi-armed chromosomes that have evolved probably by fusions of two ancestral microchromosomes, besides macrochromosomes and dot-like microchromosomes. However, it is impossible to define the inter-species homologies of such small-sized bi-armed chromosomes based on chromosome morphology and banding characteristics. Although painting probes from the chicken (Gallus gallus, GGA) chromosomes 1-9 and Z have been widely used to investigate avian chromosome homologies, GGA microchromosome probes are rarely used in these studies because most GGA microchromosome probes generated by flow sorting often contain multiple GGA microchromosomes. In contrast, the stone curlew (Burhinus oedicnemus, BOE, Charadriiformes) has an atypical low diploid chromosome number (42) karyotype and only 4 pairs of dot-like microchromosomes; a set of chromosome-specific painting probes that cover all BOE chromosomes has been generated. To get a genome-wide view of evolutionary chromosomal rearrangements in different lineages of Ciconiiformes, we used BOE painting probes instead of GGA painting probes to analyze the karyotypes of three ciconiiform species belonging to two different families: the eastern grey heron (Ardea cinerea, $\mathrm{ACl}, 2 \mathrm{n}=64$, Ardeidae), the little egret (Egretta garzetta, EGA, $2 n=64$, Ardeidae) and the crested ibis (Nipponia nippon, NNI, 2n $=68$, Threskiornithidae).

Results: BOE painting probes display the same hybridization pattern on chromosomes of $\mathrm{ACl}$ and $\mathrm{EGA}$, while a different hybridization pattern is observed on chromosomes of NNI. BOE autosome probes detected 21 conserved homologous segments and 5 fusions on the sixteen pairs of recognizable chromosomes of ACl and EGA, while 16 conserved homologous segments and 4 fusions were found on the twelve pairs of recognizable chromosomes of NNI. Only a portion of smaller bi-armed chromosomes in the karyotypes of the ciconiiform species could have evolved from fusions of ancestral microchromosomes. In particular BOE 5, which is the result of a fusion between two
\end{abstract}

\footnotetext{
*Correspondence: fengtang.yang@gmail.com; whnie@mail.kiz.ac.cn

1 State Key Laboratory of Genetic Resources and Evolution, Kunming

Institute of Zoology, Chinese Academy of Sciences, Kunming 650223,

Yunnan, People's Republic of China

${ }^{4}$ School of Life Sciences and Medicine, Shandong University

of Technology, Zibo 255049, Shandong, People's Republic of China

Full list of author information is available at the end of the article
} original author(s) and the source, provide a link to the Creative Commons licence, and indicate if changes were made. The images or other third party material in this article are included in the article's Creative Commons licence, unless indicated otherwise in a credit line to the material. If material is not included in the article's Creative Commons licence and your intended use is not permitted by statutory regulation or exceeds the permitted use, you will need to obtain permission directly from the copyright holder. To view a copy of this licence, visit http://creativecommons.org/licenses/by/4.0/. The Creative Commons Public Domain Dedication waiver (http://creativeco mmons.org/publicdomain/zero/1.0/) applies to the data made available in this article, unless otherwise stated in a credit line to the data. 
segments homologous to GGA 7 and 8 respectively, was retained also as either a single chromosome in $A C l(A C l 5)$ and $E G A$ (EGA 5) or had fused with a part of the BOE 10 equivalent in NNI (NNI 5).

Conclusion: Our painting results indicate that different chromosome rearrangements occur in different ciconiiform lineages. Some of the small-sized bi-armed chromosomes in ACI, EGA and NNI are derived from the fusions of two microchromosomes, indicating that microchromosome fusions play an important role in ciconiiform chromosome evolution. The fusion segment homologous to GGA 7 and 8 is a potential cytogenetic signature that unites Ardeidae and Threskiornithidae.

Keywords: Chromosome painting, Stone curlew, Herons, Ibises, Chromosomal rearrangements, Microchromosome fusion

\section{Background}

According to traditional classification, the order Ciconiiformes comprises medium- to large-sized wading birds that have a worldwide distribution in temperate, subtropical, and tropical regions. There are 108 species in Ciconiiformes, belonging to three suborders, 5 families and 36 genera [1]. The five families include Ardeidae (herons, bitterns, and egrets), Balaenicipitidae (shoebills), Ciconiidae (storks), Scopidae (hammerkops), and Threskiornithidae (ibises and spoonbills). Up to the year of 2000, the karyotypes of more than twenty species in Ciconiiformes, have been reported consecutively [2-17]. Only the monotypic family Scopidae (hammerkops) has not been studied. The diploid numbers of ciconiiform species studied so far vary from 52 to 78 . As found in most avian, the karyotypes of ciconiiform species are also composed of two groups of chromosomes that differ notably in size: macro- and microchromosomes (reviewed in [18, 19]). After comparing the morphology of the first three pairs of macrochromosomes in ciconiiform karyotypes, it was proposed that centric fission, pericentric inversion and centric fusion play a role in the karyotype evolution of Ciconiiformes [7, 16]. The first three macrochromosomes in Ciconiidae and Balaenicipitidae have retained their ancestral morphology, identical to that found in many other birds. The submetacentric third pair of macrochromosomes is the unique character for Ardeidae (which is subtelocentric in all other Ciconiiformes). The centric fission of the first pair of macrochromosomes was found only in Threskiornithidae. In addition, different numbers of medium to small-sized bi-armed chromosomes exist in species from different ciconiiform families [7]. However, the origin of these small-sized bi-armed chromosomes, and their inter-specific and inter-family homologies wait to be elucidated.

Cross-species chromosome painting has been utilized to detect chromosomal homologies between different avian species for more than two decades. Up to 2019, 96 avian species from eighteen orders have been analyzed by chromosome painting mainly using probes from the chicken (Gallus gallus, GGA) macrochromosomes
1-9 and Z, and four sets of probes from other bird species (reviewed in [20]). These molecular cytogenetic data have provided support for the suggestion that most avian species have an apparently conserved karyotype, only few rearrangements have occurred during about 100 million years of avian evolution [18, 21]. Comparative cytogenetic analyses have defined these evolutionary rearrangements as chromosomal fusions, fissions and inversions. These provide new insights into avian karyotype evolution and phylogenetic relationships (reviewed in [19, 22]). However, considering the huge number of extant avian species, only about $1 \%$ avian species have been analyzed by chromosome painting. Even in some orders, just two or three species have molecular cytogenetic data (reviewed in [20, 22]). For instance, in Ciconiiformes, only two storks have been studied recently using probes from GGA and Leucopternis albicollis (LAL) macrochromosomes [23], while species from the other four ciconiiform families have not been studied by chromosome painting.

The eastern grey heron (Ardea cinerea, ACI), the little egret (Egretta garzetta, EGA) and the crested ibis (Nipponia nippon, NNI) are species from two different ciconiiform families: the former two belong to the Ardeidae, the last is a member of the Threskiornithidae. Previous comparative cytogenetic study indicated that both Ardeidae and Threskiornithidae constitute a clear-cut group based on changes in chromosome morphology; however, such chromosomal changes could not provide information on the relationships between these families due to their potential independent origins and the presence of undefined small chromosomes involved in the fusion with a segment of chromosome 1 [7]. In avian cross-species chromosome painting studies, the most widely used painting probes are derived from GGA macrochromosomes 1-9 and Z, which only cover part of the avian genome, making it impossible to define the rearrangements involving microchromosomes. GGA microchromosomes are numerous and similar in size, it is difficult to generate chromosome-specific probes of single GGA microchromsomes using flow sorting and 
microdissection. While some GGA microchromosome painting probes are available, they are scarcely used in avian chromosome painting studies due to each containing multiple GGA microchromosomes. To study avian microchromosome evolution, the painting probes that cover the entire genome, including microchromosomes, of a given avian species are required. Probes from the stone curlew (Burhinus oedicnemus, BOE, $2 \mathrm{n}=42$, Charadriiformes) were the first set of chromosomespecific painting probes from Neoaves species that were able to cover the entire genome of a given bird species [24]. Thus, BOE painting probes have been used to establish genome-wide chromosomal homology among the karyotypes of eleven avian species in eight different orders [24-28]. The results demonstrate that BOE painting probes are robust tools for delineating avian chromosome evolution, especially for revealing the rearrangements that involve microchromosomes. Moreover, avian evolution are thought to involve three major events: (1) the divergence of Palaeognathae (ratites) and Neognathae (others), (2) the divergence from the Neognathae of the Galloanserae, and (3) the divergence of the remainder of Neognathae (Neoaves) [29, 30]. GGA belongs to the branch of the second divergence, which occurred about 88 million years, while BOE, ACI, EGA and NNI belong to Neoaves, their divergences completed about 50 million years ago [31]. Thus, BOE probes are better suited for comparative cytogenetic study in Neoaves than GGA probes due to the higher hybridization efficiency when applied to Neoaves species than GGA. In the present study, $\mathrm{BOE}$ painting probes were used to detect chromosomal homology in ACI, EGA and NNI, three ciconiiform species from two different families, and to define the origin of microchromosomes in the evolution of these species. To verify some of the hybridization results of BOE probes, three paint probes from GGA and the griffon vulture (Gyps fulvus, GFU) (GGA4, GFU 16 and GFU 14+27) were used in hybridizations with ACI and NNI chromosomes. Our study provides some new insights into the karyotype evolution and phylogenetic relationships in Ciconiiformes.

\section{Results}

\section{Karyotype characteristics of ACl, EGA and NNI}

To facilitate comparisons, metaphases and karyotypes of avian species studied here are shown in Fig. 1, including $\mathrm{BOE}$, the species from which the painting probes were derived (Fig. 1a and e). The karyotypes of ACI and EGA had been investigated previously $[9,10,14,17]$. However, the chromosome numbers of these two species varied in different reports, with the diploid numbers ranging from 60 to 68, due to difficulties in determining the exact number of microchromosomes (MIC). Our chromosome counts support 64 as the modal diploid number of $\mathrm{ACI}$ (Fig. 1b) and EGA (Fig. 1c). The diploid number of NNI is 68 (Fig. 1d), as reported previously [12, 15, 32]. The karyotypes of ACI (Fig. 1f) and EGA (Fig. 1g) are similar, consisting of five pairs of large to medium-sized bi-armed chromosomes, one pair of medium-sized acrocentric chromosomes, ten pairs of medium to small-sized biarmed chromosomes and fifteen pairs of dot-like microchromosomes. The karyotype of NNI (Fig. 1h) consists of three pairs of large bi-armed chromosomes, one pair of large telocentric chromosomes, eight pairs of mediumsized to small-sized bi-armed chromosomes, and twenty-one pairs of dot-like microchromosomes. The $\mathrm{Z}$ chromosome is a medium-sized metacentric in these three species (Fig. 1f-h), while the W chromosome is a small acrocentric in EGA (Fig. 1g). In comparison with karyotypes of GGA and BOE, the karyotypes of ciconiiform species have different number of medium to smallsized bi-armed chromosomes as found in BOE (Fig. 1 a and 1e), and the number of dot-like microchromosomes is lower than that of GGA.

\section{Hybridization of $\mathrm{BOE}$ probes onto chromosomes of $\mathrm{ACl}$ and EGA}

The hybridization patterns produced by each BOE probe on metaphases of ACI and EGA are identical, confirming that ACI and EGA have similar karyotypes. Examples of BOE probes hybridizing onto chromosomes of ACI and EGA are shown in Figs. 2 and 3. The first six chromosome pairs of ACI and EGA are highly conserved, each showing homology with one BOE macrochromosome (BOE 1-6) (corresponding to GGA $1-3,4 \mathrm{q}, 7+8$ and 5) respectively (Figs. 2a and 3a). Five out of the ten smaller bi-armed chromosomes of ACI and EGA were each painted by two BOE probes and five syntenic homologous segment associations [BOE $7+10,7+11,8+10$, $9+14$ and $11+(15+16)]$ (corresponding to GGA $9+1$ $\mathrm{MIC}, 1 \mathrm{MIC}+1 \mathrm{MIC}, 1 \mathrm{MIC}+1 \mathrm{MIC}$ and $1 \mathrm{MIC}+1$ $\mathrm{MIC}$ ) are revealed in ACI (Fig. 2b-f) and EGA (Fig. 3bf). The remaining five small bi-armed chromosomes of ACI and EGA each corresponds to one BOE segment (BOE 9q, 8p, 13q, 7q and 12q) (corresponding to GGA 6, $4 \mathrm{p}$, and $3 \mathrm{MICs})$. Some dot-like microchromosomes in $\mathrm{ACI}$ and EGA are painted by one BOE probe, and some are not hybridized by any $\mathrm{BOE}$ probe. The $\mathrm{Z}$ chromosome probe of $\mathrm{BOE}$ hybridizes to the whole $\mathrm{Z}$ chromosomes of $\mathrm{ACI}$ and EGA, and the W chromosome probe of BOE paints the $\mathrm{W}$ chromosome and the distal part of the long arm of EGA Z (Zqter). The hybridization results of BOE probes on chromosomes of ACI and EGA are summarized in Figs. 4 and 5 and Table 1. 

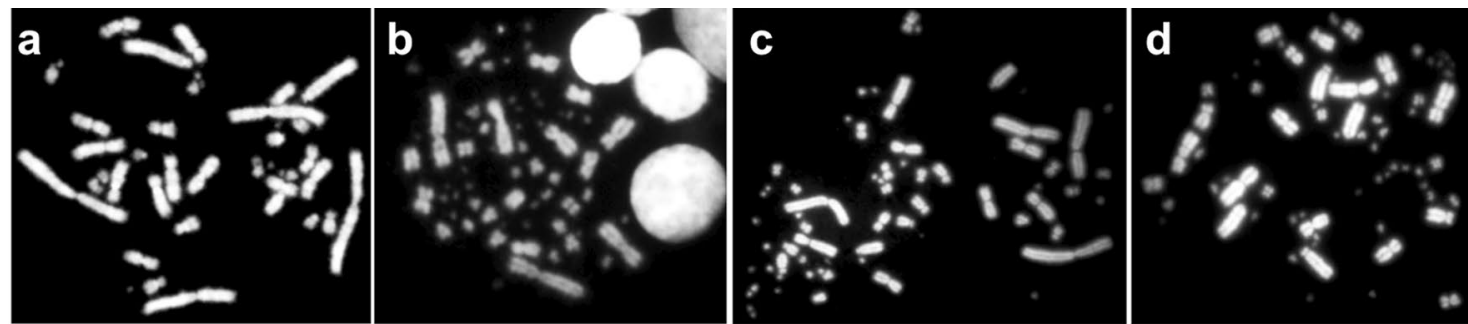

e

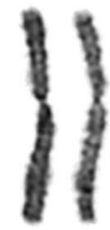

1

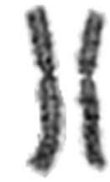

2

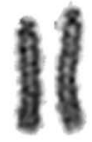

3

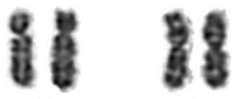

5

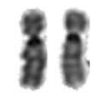

6

है

7

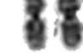

89

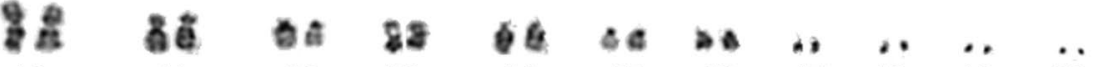

f

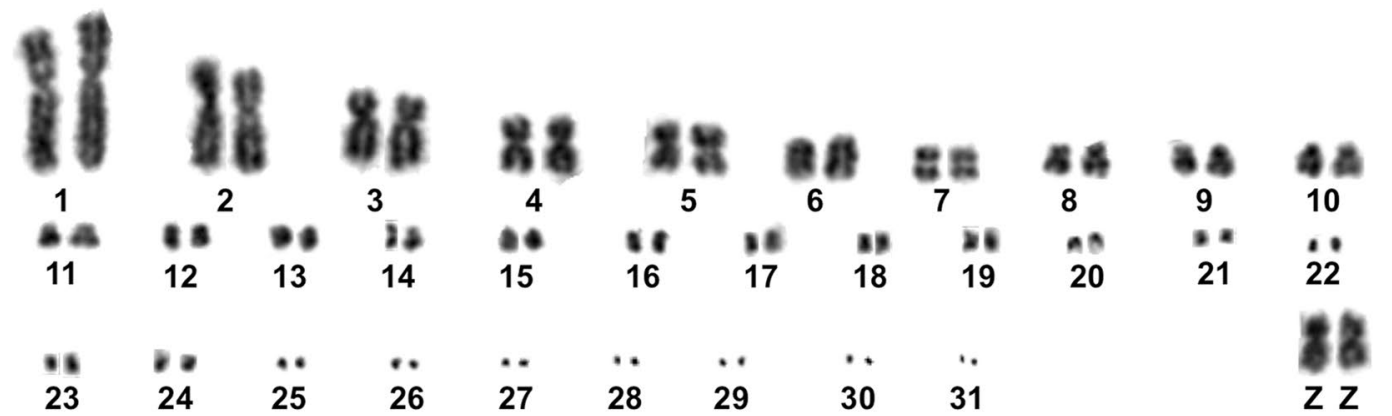

g
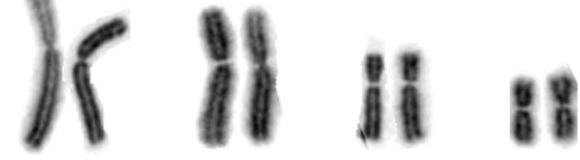

80

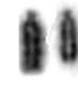

g. ถ้ ถ้

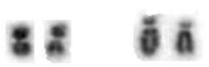

$88-58$

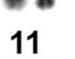

86 ถ⿱一⿻上丨 12

3

4

5

6

$7 \quad 8$

$9 \quad 10$

88 . 8 :

4. $*$ * * * ,

$\because \quad \cdots \quad$

314

15

$16 \quad 17$

$\cdots$

$$
\begin{array}{lll}
* & * & *
\end{array}
$$

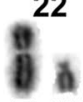

23

$24 \quad 25$

26

27

28

$29 \quad 30$

31

Z W

h

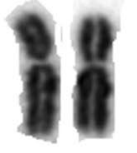

1

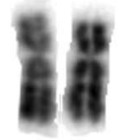

2

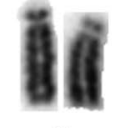

3

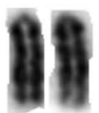

4

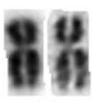

5

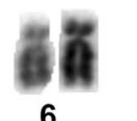

6

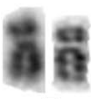

7

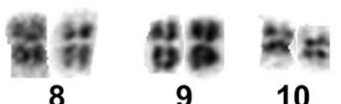

$8 \quad 9 \quad 10$

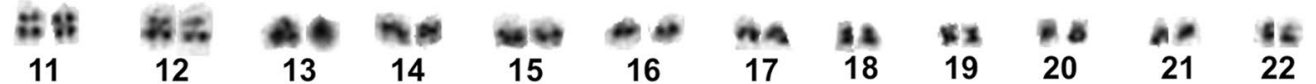

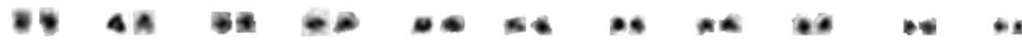
at

$\begin{array}{lllllllllll}23 & 24 & 25 & 26 & 27 & 28 & 29 & 30 & 31 & 32 & 33\end{array}$

Fig. 1 Metaphases and karyotypes of Burhinus oedicnemus ( $B O E, 2 n=42)$, Ardea cinerea ( $A C l, 2 n=64)$, Egretta garzetta $(E G A, 2 n=64)$, and Nipponia nippon $(\mathrm{NNI}, 2 \mathrm{n}=68)$ stained and banded with DAPI. a The metaphase of BOE. $\mathbf{b}$ The metaphase of ACl. $\mathbf{c}$ The metaphase of EGA. $\mathbf{d}$. The metaphase of NNI. e The karyotype of BOE. $\mathbf{f}$ The karyotype of ACl. $\mathbf{g}$ The karyotype of EGA. $\mathbf{h}$ The karyotype of NNI 


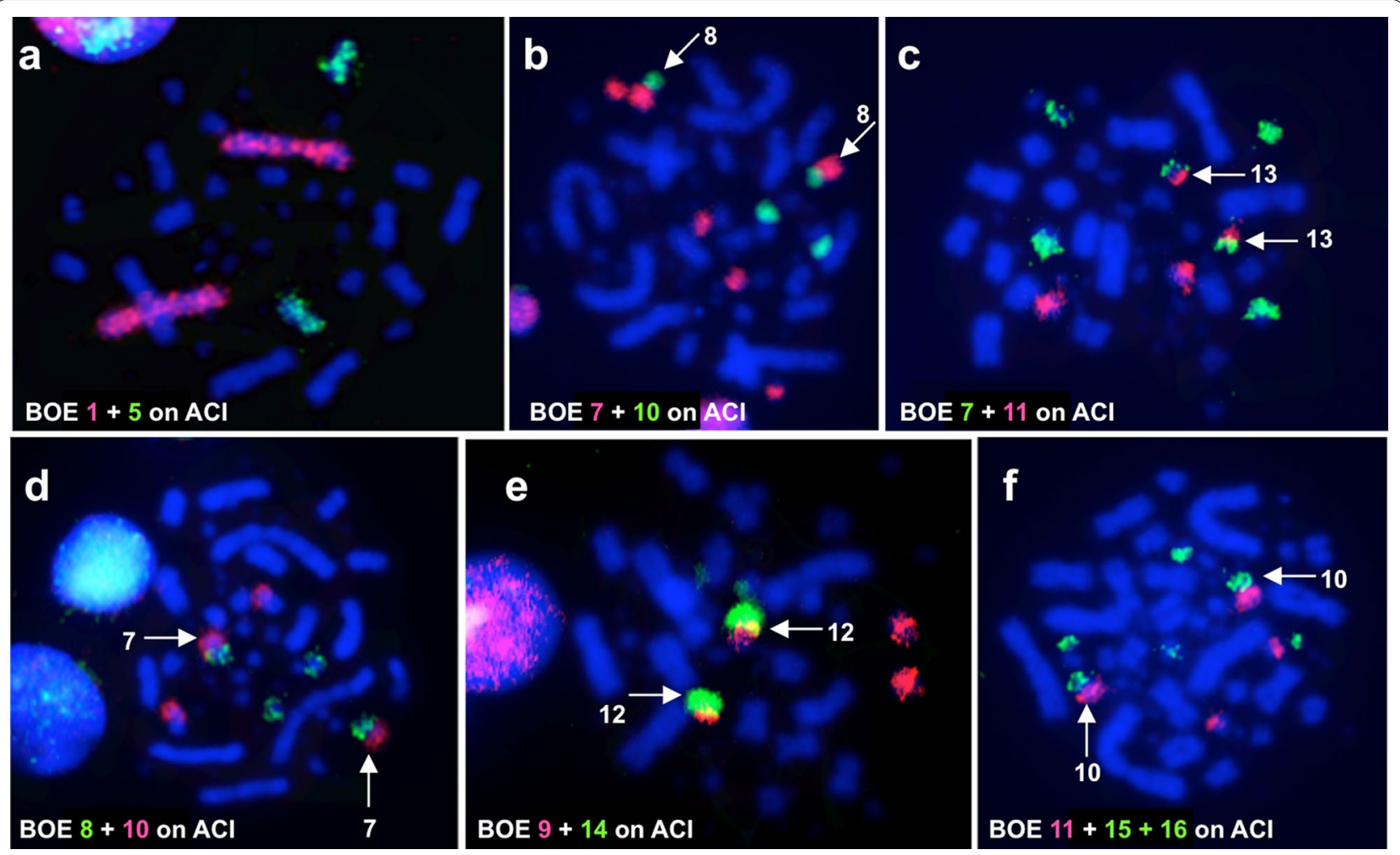

Fig. 2 Dual-color FISH examples of ACl. a Hybridization of BOE 1 (red) and 5 (green) probes to ACl chromosomes. b Hybridization of BOE 7 (red) and 10 (green) probes to ACl chromosomes, arrows show ACl 8. c Hybridization of BOE 7 (green) and 11 (red) probes to ACl chromosomes, arrows show $\mathrm{ACl}$ 13. d Hybridization of BOE 8 (green) and 10 (red) probes to ACl chromosomes, arrows show ACl 7. e Hybridization of BOE 9 (red) and 14 (green) probes to $\mathrm{ACl}$ chromosomes, arrows show $\mathrm{ACl}$ 12.f Hybridization of $\mathrm{BOE} 11$ (red) and 15+ 16 (green) probes to $\mathrm{ACl}$ chromosomes, arrows show $\mathrm{ACl} 10$

\section{Hybridization of BOE probes onto chromosomes of NNI}

Eighteen chromosome painting probes from BOE were hybridized to metaphases of NNI. All BOE probes produce distinctive signals on NNI chromosomes. FISH examples are shown in Fig. 6, and the results of chromosome painting between BOE and NNI are summarized in Fig. 7 and Table 1. The hybridization patterns of BOE probes in NNI (Fig. 7) are different from those observed in ACI (Fig. 4) and EGA (Fig. 5). A fission of BOE 1 (corresponding to GGA 1) homologue and a fusion of two BOE homologous segments (BOE 1p $+9 q$ ) (corresponding to GGA $1 p+6$ ) are detected by BOE probes in NNI (Fig. 6a). Each of four NNI macrochromosomes is homologous to one BOE chromosome (BOE 2-4, 6) (corresponding to GGA 2, 3, 4q and 5). In addition, three syntenic associations of BOE segments (BOE $5+10,7+10$ and $8+11)$ [corresponding to GGA $(7+8)+1 \mathrm{MIC}, 9+1$ $\mathrm{MIC}$ and $1 \mathrm{MIC}+1 \mathrm{MIC}$ ] were found in the karyotype of NNI (Fig. 6b-d). The three smallest bi-armed chromosomes of NNI are each homologous to one BOE segment (BOE 8p, 7qpart and 13q) (corresponding to GGA 4p+2 MICs). The pattern of hybridization results in NNI sex chromosomes and dot-like microchromosomes (Fig. 6f) are identical to the pattern shown in ACI and EGA mentioned above.

\section{Hybridization of three GGA and GFU probes onto chromosomes of $\mathrm{ACl}$ and NNI}

To clarify previous cytogenetic results and to resolve some uncertainties about the interpretation of hybridization results of $\mathrm{BOE}$ probes on the origin of microchromosomes, we selected three GGA and GFU probes (GGA 4, GFU 16 and $14+27$ ) to paint the chromosomes of ACI and NNI. Since ACI and EGA have similar karyotypes and identical hybridization patterns when painted with BOE probes, EGA is not included in this part of the study. Both ACI 3 (submetacentric) and NNI 3 (acrocentric) are painted by the probe of BOE 3 (corresponding to GGA 3) (see above). When we used the probe of GFU 16, which is homologous to part of BOE 3 [26], the distal part of the short arm of ACI 3 was not painted (Fig. 8a), while the short arm of NNI 3 was hybridized (Fig. 8b), confirming that a pericentric inversion had occurred in the ardeid ancestor. As 

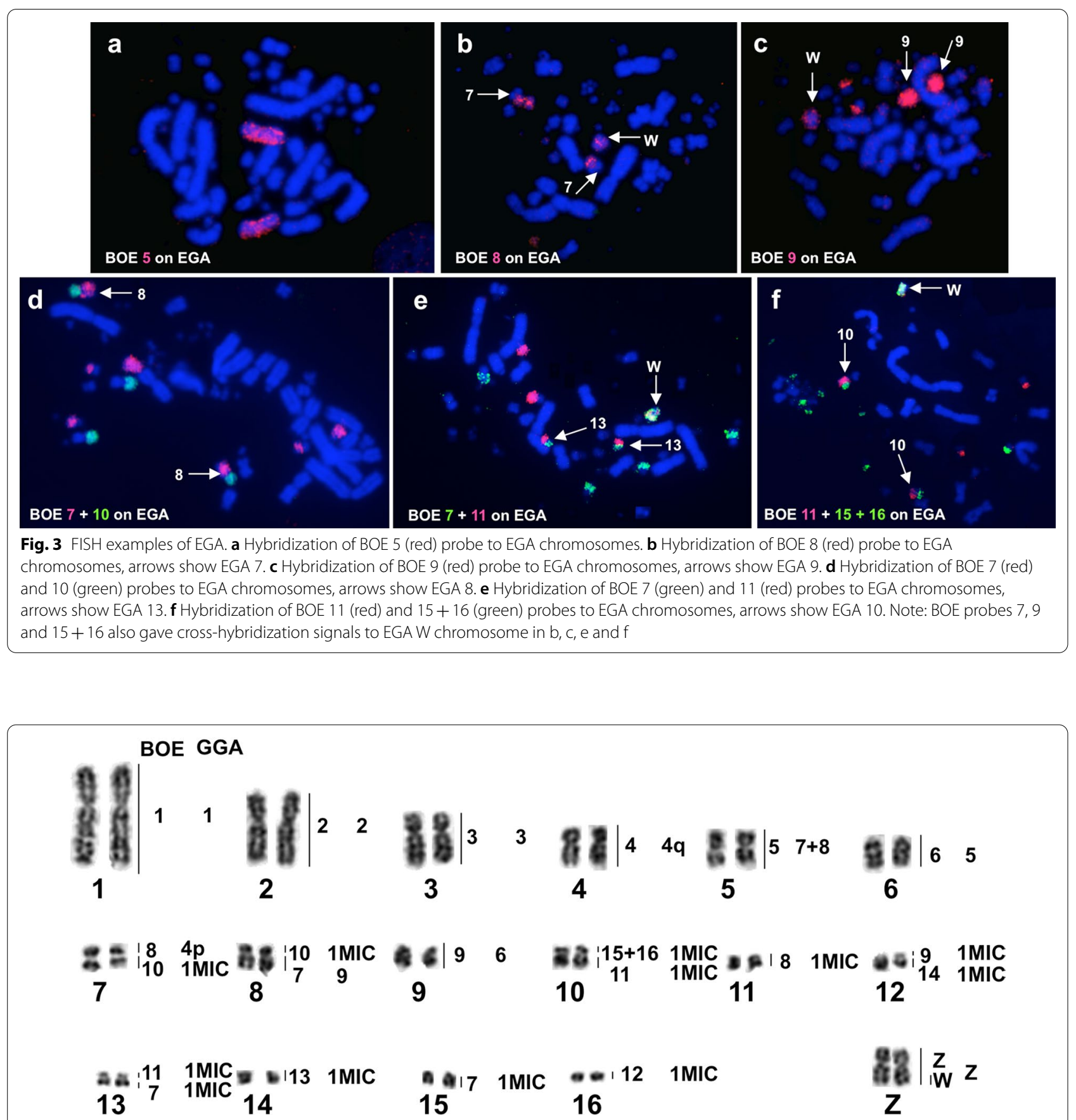

Fig. 4 Partial DAPI-banded karyotype of ACI with assignment of homologies to BOE and GGA. p: the short arm of a chromosome; q: the long arm of a chromosome; MIC: microchromosome

mentioned above, BOE 8 and 10 probes each painted two segments of ACI and NNI chromosomes respectively, but we cannot determine the regional origin of each of these two homologous segments using the BOE whole chromosome paints. Previous studies indicated that GFU 27 was homologous to the short arm of BOE 10 (BOE 10p), and the short arm of GGA 4 (GGA 4p) corresponded to the short arm of BOE 8 (BOE 8p) [24, 26]. The hybridization of GFU 27 (sorted together with GFU 14) probe to chromosomes of ACI and NNI indicate that ACI 8p was homologous to BOE 10p (Fig. 8c), while the distal part of the short arm of NNI 5 was homologous to BOE 10p (Fig. 8d). As found in most avian species, the GGA 4 probe gave two signals on ACI 


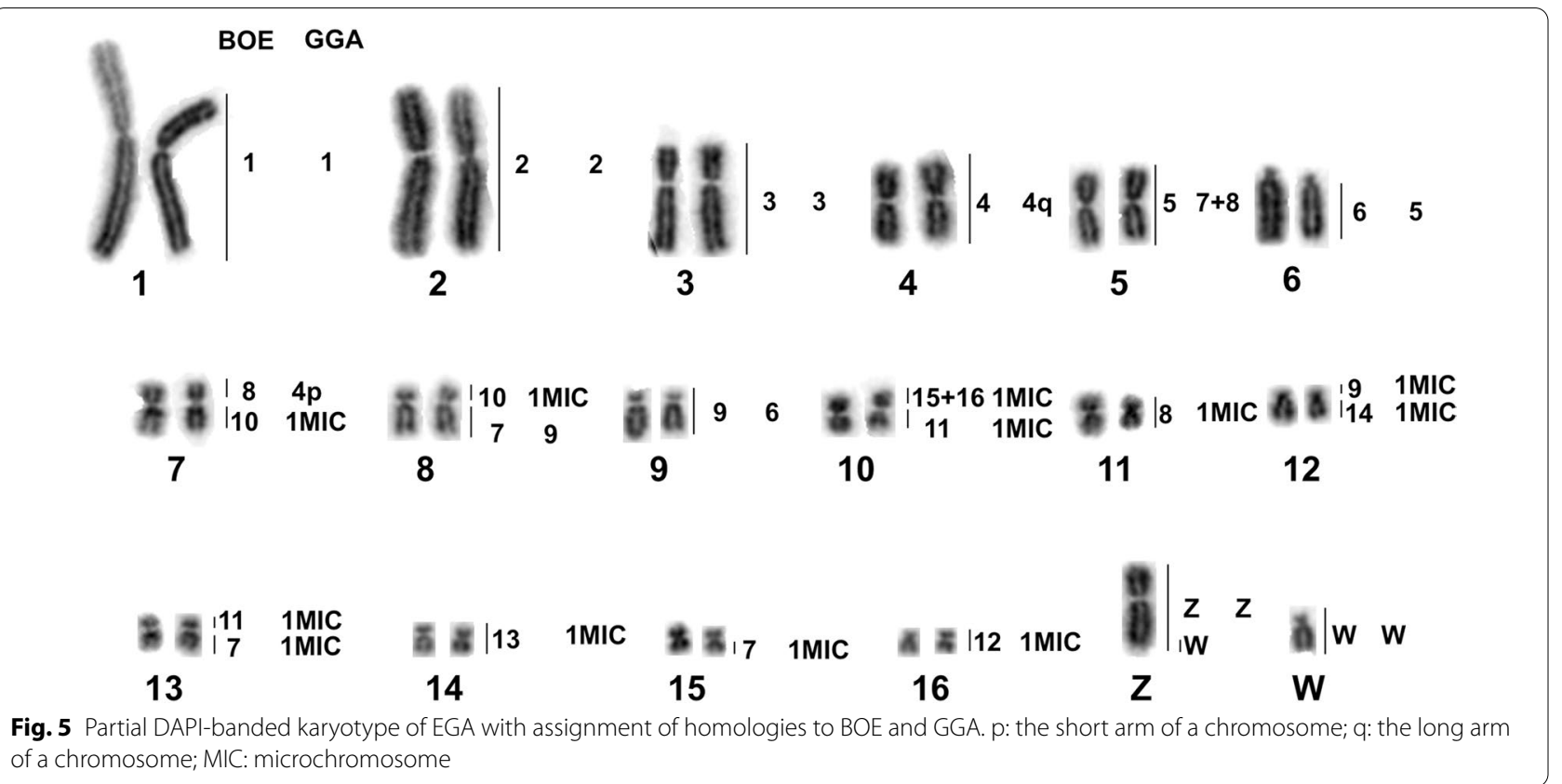

Table 1 Chromosomal correspondence between Burhinus oedicnemus (BOE), Gallus gallus (GGA), Ardea cinerea (ACl), Egretta garzetta (EGA) and Nipponia nippon (NNI) revealed by chromosome painting with BOE chromosome-specific painting probes

\begin{tabular}{lllll}
\hline BOE & GGA & ACI & EGA & NNI \\
\hline 1 & 1 & 1 & 1 & $2 q, 4$ \\
2 & 2 & 2 & 2 & 1 \\
3 & 3 & 3 & 3 & 3 \\
4 & $4 q$ & 4 & 4 & 6 \\
5 & 7,8 & 5 & 5 & $5 p+q$ (part) \\
6 & 5 & 6 & 6 & 7 \\
7 & 9,2 MICs & $8 q, 13 q, 15 q$ & $8 q, 13 q, 15 q$ & $8 q, 11,1$ MIC \\
8 & $4 p, 1$ MIC & $7 p, 11$ & $7 p, 11, W$ & $9 p, 10$ \\
9 & 6,1 MIC & $9,12 p$ & $9,12 p, W$ & $2 p, 1$ MIC \\
10 & 2 MICs & $7 q, 8 p$ & $7 q, 8 p, W$ & $5 q$ ter, $8 p$ \\
11 & 2 MICs & $10 q, 13 p$ & $10 q, 13 p$ & $9 q, 1$ MIC \\
12 & 2 MICs & 16,1 MIC & 16,1 MIC, W & 12,1 MIC \\
13 & 2 MICs & 14,1 MIC & 14,1 MIC, W & 2 MICs, W \\
14 & 2 MICs & $12 q, 1$ MIC & $12 q, 1$ MIC & 2 MICs \\
15,16 & 3 MICs & $10 p, 2$ MICs & $10 p, 2$ MICs, W & 3 MICs, Zpter \\
$17,18,19,20$ & 1 MIC & $\sim 4$ MICs & $\sim 4$ MICs & $\sim 4$ MICs \\
Z & Z & Z & Z,W & Z, W \\
W & W, Zqter & Zqter & W, Zqter & W, Zqter \\
\hline
\end{tabular}

MIC microchromosome; $p$ the short arm of a chromosome; $q$ the long arm of a chromosome; pter the distal of the short arm of a chromosome; qter the distal of the long arm of a chromosome and NNI chromosomes. GGA4p was homologous to one smaller chromosome of ACI (ACI 11, Fig. 8e) and NNI (NNI 10, Fig. 8f) respectively. These results clarify the above uncertainties using BOE probes and establish the chromosomal correspondence among BOE, GGA, ACI, EGA and NNI (Table 1 and Figs. 4, 5 and 7).

\section{Discussion}

Previous studies have indicated that five pairs of $\mathrm{BOE}$ macrochromosomes (BOE 1-4, 6) are highly conserved. As found in many other avian species, the five largest ancestral avian chromosomes, homologous to GGA 1-3, $4 \mathrm{q}$ and 5 , are each retained as a single intact BOE chromosome (BOE 1-4 and 6), while the other 10 pairs of BOE bi-armed chromosomes (BOE 5, 7-15) each represent a fusion product of two or three homologous GGA smaller macrochromosomes or microchromosomes [24]. Therefore, probes from medium-sized BOE chromosomes are ideal tools for studying evolutionary rearrangements involving microchromosomes in different avian species. Recently, bacterial artificial chromosome (BAC) probes from GGA and zebra finch (Taeniopygia guttata, Passeriformes) have been applied to investigate the chromosomal evolution of 41 species in 16 different orders [28, 33-41]. These studies indicate that most bird species so far studied have maintained an evolutionary stability in their microchromosome organization, except for species in a few orders (e.g., Falconiformes, Psittaciformes, Cuculiformes, and Suliformes), in which microchromosome fusions have been detected. In our study, microchromosome fusions were also observed 

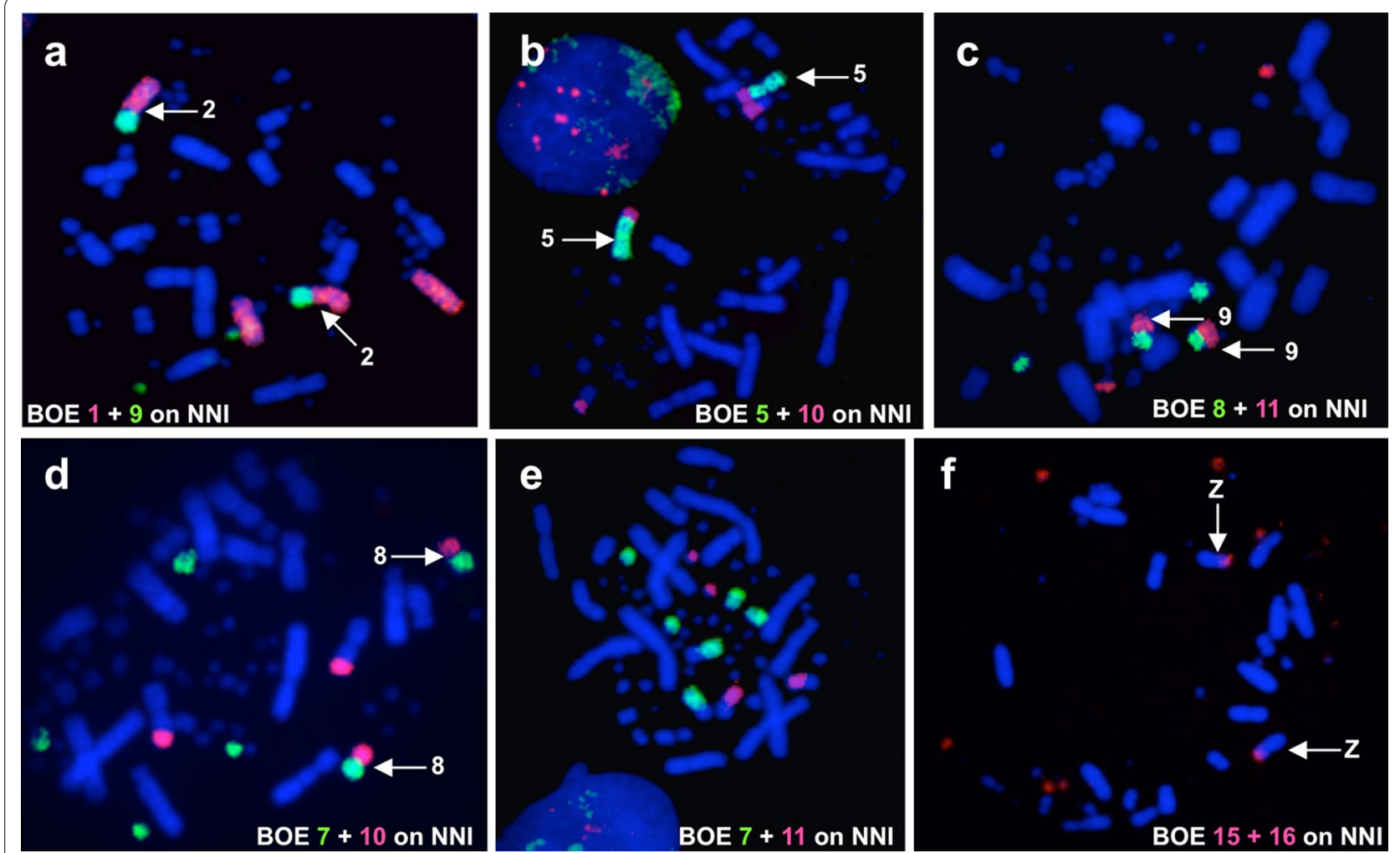

Fig. 6 FISH examples of NNI. a Hybridization of BOE 1 (red) and 9 (green) probes to NNI chromosomes, arrows show NNI 2. $\mathbf{b}$ Hybridization of BOE 5 (green) and 10 (red) probes to NNI chromosomes, arrows show NNI 5. c Hybridization of BOE 8 (green) and 11 (red) probes to NNI chromosomes, arrows show NNI 9. d Hybridization of BOE 7 (green) and 10 (red) probes to NNI chromosomes, arrows show NNI 8. e Hybridization of BOE 7 (green) and 11 (red) probes to NNI chromosomes. f Hybridization of BOE $15+16$ (red) to NNI chromosomes, arrows show NNI Z chromosomes with cross-hybridization signals

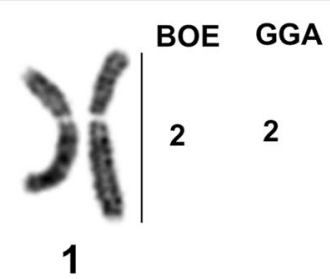

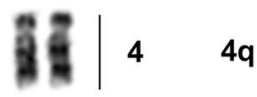

6

리

11

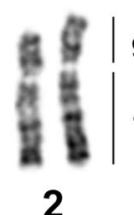

2

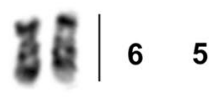

7

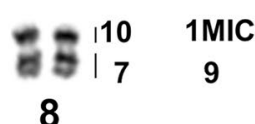

8

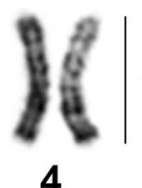

4

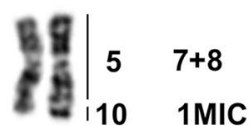

5

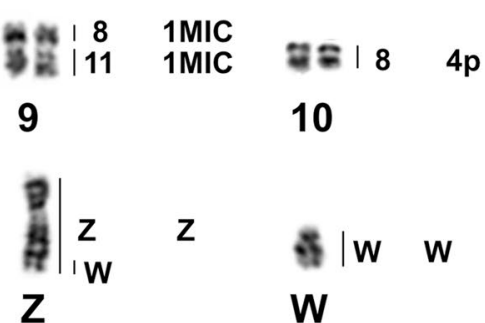

Fig. 7 Partial DAPI-banded karyotype of NNI with assignment of homologies to BOE and GGA. p: the short arm of a chromosome; q: the long arm of a chromosome; MIC: microchromosome 

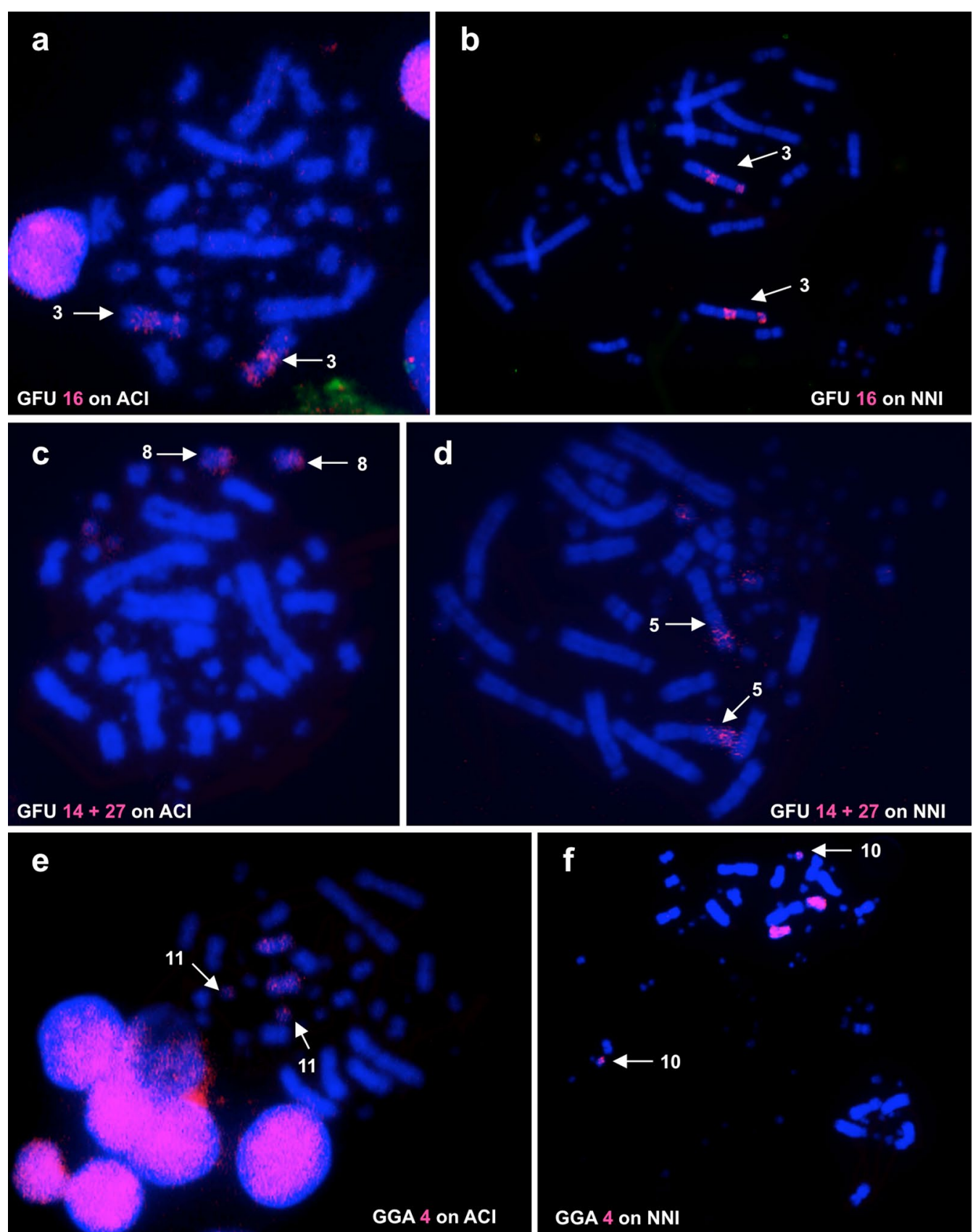

Fig. 8 Chromosome painting using selected GGA and GFU probes to hybridize to chromosomes of ACl and NNI. a Hybridization of GFU 16 probe (red) to ACl chromosomes, arrows show ACl 3. b Hybridization of GFU 16 (red) probe to NNI chromosomes, arrows show NNI 3. c Hybridization of GFU 27 (red) probe (containing GFU 14) to ACl chromosomes, arrows show ACl 8. d Hybridization of GFU 27 (red) probe (containing GFU 14) to NNI chromosomes, arrows show NNI 5. e Hybridization of GGA 4 probe (red) to ACl chromosomes, arrows show ACl 11. f Hybridization of GGA 4 probe (red) to NNI chromosomes, arrows show NNI 10

in three ciconiiform species by using BOE probes, adding Ciconiiformes to this list of exceptions. Our results demonstrate that $\mathrm{BOE}$ probes and $\mathrm{BAC}$ mapping are alternative approaches for revealing avian microchromosome fusions. Although we failed to establish the complete microchromosome correspondences between $\mathrm{BOE}$ and the three ciconiiform species due to the difficulty in identifying avian dot-like microchromosomes, the BOE painting results in other avian species indicate the number of microchromosomes painted and the 
quantity of microchromosome fusions. We expect that more avian species will be studied by using BOE probes in the future.

Up to now, the karyotypes of five species in three ciconiiform families have been studied by chromosome painting using probes from GGA, LAL and BOE ([23], this study). Since the comparative chromosome map between BOE and GGA has been established [24], the chromosome correspondence among GGA, BOE and other avian species can be inferred. Despite the use of different probes in these studies, using GGA chromosome homology as the reference, the chromosomal rearrangements found in this study can be compared with those in other ciconiiform species and can shed light on their evolutionary relationships. Table 2 lists the homologous correspondence between GGA 1-9 and their counterparts in $\mathrm{BOE}$ and the five ciconiiform species so far studied. These data indicate that species from different ciconiiform families have experienced different chromosome rearrangements during evolution. For example, different numbers of chromosomal fusions (3, 5 and 6 ) have been detected in Ciconiidae, Ardeidae and Threskiornithidae.

Among the fusions detected in Ardeidae and Threskiornithidae, two (corresponding to GGA $7+8$ and $9+1 \mathrm{MIC}$ ) seem to be common for species in our study. BOE 5 (corresponding to GGA $7+8$ ) as a conserved synteny was detected in ACI (Fig. 2a), EGA (Fig. 3b) and NNI. Furthermore, the BOE 5 homologous segment was fused to one homologous segment from part of BOE 10 (corresponding to one GGA MIC) to form NNI 5 (Fig. 6b). Besides these three ciconiiform species, to date this association of GGA $7+8$ is found only in BOE and the Southern lapwing (Vanellus chilensis), two species belonging to Charadrii which is one of the three major clades of Charadriiformes [24, 42]. Including BOE and $V$. chilensis, five species in Charadriiformes have been studied by chromosome painting; this association is not detected in the other three species of Charadriiformes [25, 27, 43]. Therefore, the GGA $7+8$ fusion could have happened independently in Charadrii and the three ciconiiform species since these lineages (Charadriiformes and Ciconiiformes) are distantly related according to phylogenetic studies [30, 31, 44]. This fusion could be a common cytogenetic character linking Ardeidae with Threskiornithidae. The fusion of BOE $7+10$ (corresponding to GGA $9+1 \mathrm{MIC}$ ) was found in ACI (Fig. 2b), EGA (Fig. 3d) and NNI (Fig. 6d), but our painting results with the probe of GFU 27 (corresponding to BOE 10p) indicate that this is not a true common character for Ardeidae and Threskiornithidae, because the microchromosome fused to the GGA 9 homologue is different in these three species (Fig. 8c and d).

In Ciconiidae, three fusions (corresponding to GGA $8+9,6+1 \mathrm{MIC}$ and $4 \mathrm{p}+1 \mathrm{MIC}$ ) were observed in two Brazilian stork species, and one of the fusions (corresponding to GGA $8+9$ ) was considered to be a shared character for storks [23]. In our study, the fusions of GGA $8+9$ and $6+1$ MIC were not detected in the three ciconiiform species, while the painting results using the GGA 4 probe indicated that GGA $4 \mathrm{p}$ was homologous to one whole microchromosome in $\mathrm{ACI}$ (Fig. 8e) and NNI (Fig. 8f) and not included in a fusion. No shared fusions have been found yet in the species of Ciconiidae and other ciconiiform families (Ardeidae and Threskiornithidae).

Although only five ciconiiform species have been studied by chromosome painting to date, these results demonstrate that storks differ from herons and ibises in chromosome rearrangements, and herons and ibises share a syntenic association that indicates a closer karyotype relationship. These chromosome painting data also provide support for the molecular phylogenomic studies that place herons and ibises in one clade and storks in another clade [30, 44-49]. To further elaborate the karyotype relationships among different

Table 2 Homologies between GGA (G. gallus) 1-9 and chromosomes of BOE and five species in Ciconiiformes revealed by chromosome painting

\begin{tabular}{|c|c|c|c|c|c|c|c|c|c|c|c|}
\hline \multirow[t]{2}{*}{ Species } & \multirow[t]{2}{*}{$2 n$} & \multicolumn{9}{|c|}{ Homologues of GGA 1-9 } & \multirow[t]{2}{*}{ References } \\
\hline & & 1 & 2 & 3 & 4 & 5 & 6 & 7 & 8 & 9 & \\
\hline Burhinus oedicnemus, BOE & 42 & 1 & 2 & 3 & $4,8 p$ & 6 & $9 q$ & $5 q$ & $5 p$ & $7 p$ & [1324] \\
\hline Ardea cinerea, ACl & 64 & 1 & 2 & 3 & 4,11 & 6 & 9 & $5 q$ & $5 p$ & $8 q$ & This study \\
\hline Egretta garzetta, EGA & 64 & 1 & 2 & 3 & 4,11 & 6 & 9 & $5 q$ & $5 p$ & $8 q$ & This study \\
\hline Nipponia nippon, NNI & 68 & $2 \mathrm{q}, 4$ & 1 & 3 & 6,10 & 7 & $2 p$ & $5 q$ & $5 p$ & $8 q$ & This study \\
\hline Jabiru mycteria, JMY & 56 & 1 & 2 & 3 & $4,8 q$ & 5 & $6 p$ & 9 & $7 q$ & $7 p$ & {$[23]$} \\
\hline Ciconia maguari, CMA & 72 & 1 & 2 & 3 & $4,8 q$ & 5 & $7 q$ & 9 & $6 q$ & $6 p$ & {$[23]$} \\
\hline
\end{tabular}

$p$ the short arm of a chromosome; $q$ the long arm of a chromosome 
ciconiiform families, chromosome painting data are needed from more ciconiiform species and from Pelecaniformes, an order which shows some karyotypic similarities and a closer phylogenetic relationship with Ciconiiformes.

\section{Conclusions}

We have established genome-wide comparative chromosome maps between $\mathrm{BOE}$ and three ciconiiform species from two different families by chromosome painting using a set of BOE probes that cover the entire BOE genome. Our results indicate that chromosomal fusions play an important role in the karyotype evolution of species in Ciconiiformes. Besides fusions between macro- and microchromosome, fusions between microchromosomes are also found in species from Ardeidae and Threskiornithidae, leading to some small bi-armed chromosomes. A fusion of segments homologous to GGA 7 and 8 is shared by these three ciconiiform species, indicating that this fusion may be a cytogenetic signature linking Ardeidae and Threskiornithidae, and reinforcing the proposition that places herons and ibises in one clade as reported in previous molecular phylogenomic studies.

\section{Methods}

\section{Cell culture, metaphase preparation and chromosome nomenclature}

Three species belonging to two different families in Ciconiiformes, the eastern grey heron (A. cinerea, ACI), the little egret (E. garzetta, EGA) and the crested ibis $(N$. nippon, NNI) were studied. The fibroblast cell lines of EGA (KCB 2011136, $q$ and 2012017, ఠ) and NNI (KCB 2011119, o and 2012055S, \&) were provided by Kunming Cell Bank, the Chinese Academy of Sciences. The cells were grown in DMEM (Dulbecco's Modified Eagle Medium) enriched with $15 \%$ fetal bovine serum. Metaphase preparation and slide preparation of NNI and EGA were carried out following conventional methods. Mitotic chromosomes of ACI were obtained from the bone marrow suspension of a male ACI prepared and stored in 1999 in Kunming Cell Bank, the Chinese Academy of Sciences.

The karyotype of ACI and EGA were arranged according to the relative length of the chromosomes from the largest to the smallest. The chromosomes of NNI were numbered according to previously published karyotypes with a minor revision $[12,15,32]$.

\section{FISH, image capture and processing}

Painting probes from the stone curlew (B. oedicnemus, $\mathrm{BOE}$ ) were hybridized onto metaphases of ACI, EGA and NNI respectively to delineate the homologous segments between BOE, ACI, EGA and NNI. In addition, three painting probes from the chicken (G. gallus, GGA) and the griffon vulture (G. fulvus, GFU) were used to verify results from BOE probes. The preparation of BOE, GGA and GFU painting probes, crossspecies chromosome painting, image capture and processing were carried out as previously described $[24,26]$. In brief, the whole set of BOE painting probes and part of GFU and GGA painting probes were generated from flow-sorted chromosomes and amplified by degenerate oligonucleotide-primed polymerase chain reaction (DOP-PCR). Painting probes was labeled with either Biotin-16-dUTP or digoxigenin-11-dUTP (Roche, Basel, Switzerland) in a second round of PCR amplification and visualized using $\mathrm{Cy} 3$-avidin (Amersham), mouse anti-digoxigenin monoclonal antibody (Sigma, D8156) and goat anti-mouse FITC conjugate antibody (Sigma, F0257). Hybridization signals (homologous to BOE chromosomes or chromosomal segments) were assigned to specific chromosomes or chromosome regions of ACI, EGA and NNI defined by inverted DAPI-banding patterns. After single color FISH, dual-color FISH was performed to further verify associations between different conserved segments in the rearranged chromosomes of ACI, EGA and NNI. To facilitate comparison of homologous chromosomal segments across bird species, the homologous GGA chromosome segments as inferred from the GGA-BOE comparative chromosomal map [24] were indicated beside ACI, EGA and NNI chromosomes.

\section{Abbreviations \\ GGA: Gallus gallus; LAL: Leucopternis albicollis; ACl: Ardea cinerea; EGA: Egretta garzetta; NNI: Nipponia nippon; BOE: Burhinus oedicnemus; 2n: Diploid number; GFU: Gyps fulvus; MIC: Microchromosome; DAPI: 4', 6-Diamidino-2-phenylin- dole; BAC: Bacterial artificial chromosome; JMY: Jabiru mycteria; CMA: Ciconia maguari; KCB: Kunming cell bank; DMEM: Dulbecco's Modified Eagle Medium; FISH: Fluorescence in situ hybridization; p: The short arm of a chromosome; q: The long arm of a chromosome.}

\section{Acknowledgements}

We would like to thank Prof. Shuguang Wei and Ms. Rong Su, Key Laboratory of Forensic Sciences, Ministry of Health, Xi'an Jiaotong University, for their help with the experiments.

\section{Authors' contributions}

JW participated in designing the study, performed cell culture and chromosome painting probes labeling and data analysis, drafted and revised the manuscript. WS and YH performed cell culture, chromosome preparation and chromosome painting experiments. SL provided materials for KCB to establish cell lines of EGA and NNI, and critical review of the manuscript for important intellectual content. PCMO and MAFS contributed to the chromosome sorting and the manuscript revision. FY contributed to the study design, data analysis and the manuscript revising. WN participated in designing the study, performing chromosome painting experiments, analyzing data, drafting and revising the manuscript. All the authors read and approved the final manuscript. 


\section{Funding}

This work was partly supported by grants from the Ministry of Science and Technology of China (NSTI-BMCR20), Chinese Academy of Sciences (CZBZX-1) and Animal Branch of the Germplasm bank of wild species in Southwest China, CAS (Large Research infrastructure Funding).

\section{Availability of data and materials}

All data supporting the results reported in this article can be found at the article itself. No additional dataset is available.

\section{Declarations}

\section{Ethics approval and consent to participate}

All experiments were performed in accordance with the relevant national and international guidelines and approved by the Institutional Animal Care and Use Committee, Kunming Institute of Zoology, Chinese Academy of Sciences.

\section{Consent for publication}

Not applicable.

\section{Competing interests}

The authors declare that they have no competing interests.

\section{Author details}

${ }^{1}$ State Key Laboratory of Genetic Resources and Evolution, Kunming Institute of Zoology, Chinese Academy of Sciences, Kunming 650223, Yunnan, People's Republic of China. ${ }^{2}$ Key Laboratory of Forensic Sciences, Ministry of Health, Xi'an Jiaotong University, Xi'an 710061, People's Republic of China. ${ }^{3}$ Cambridge Resource Centre for Comparative Genomics, Department of Veterinary Medicine, University of Cambridge, Cambridge CB3 OES, UK. ${ }^{4}$ School of Life Sciences and Medicine, Shandong University of Technology, Zibo 255049, Shandong, People's Republic of China.

\section{Received: 25 October 2021 Accepted: 18 February 2022}

Published online: 03 March 2022

\section{References}

1. Mayr E, Cottrell G. Check-list of birds of the world. Revision of the work of James L. Peters. 2nd ed. Cambridge: Harvard University Press; 1979.

2. Beltermam RHR, de Boer LEM. A miscellaneous collection of bird karyotypes. Genetica. 1990;83:17-29.

3. Belterman RHR, De Boer LEM. A karyological study of 55 species of birds, including karyotypes of 39 species new to cytology. Genetica. 1984:65:39-82.

4. Bian X, Cai H, Ning S, Xong X, Han L, Chen X, Liu A, Yang L. Studies on the karyotypes of birds XII.15 Species of nonpasserines (Aves). Zool Res. 1991;12:407-12

5. Bian X, Li Q, Zhang H. Karyotypes of eight species of birds in Ciconiiformes and Gruiformes. Chin J Zool. 1990;25:11-5.

6. de Boer LEM. New developments in vertebrate cytotaxonomy VIII. A current list of references on avian karyology. Genetica. 1984;65:3-37.

7. de Boer LEM, van Brink JM. Cytotaxonomy of the Ciconiiformes (Aves), with karyotypes of eight species new to cytology. Cytogenet Cell Genet. 1982;34:19-34

8. Francisco MR, Junior PMJ. First karyotypical description of two American Ciconiiform birds, Mycteria americana (Ciconiidae) and Platalea ajaja (Threskiornithidae) and its significance for the chromosome evolutionary and biological conservation approaches. Genetics Mol Biol. 2000;23:799-801.

9. Hammar B. The karyotypes of thirty-one birds. Hereditas. 1970;65:29-58.

10. Itoh N, Ikeuehi T, Shimba H, Mori M, Sasaki M, Makino S. A comparative karyotype study in fourteen species of birds. Jpn J Genetics. 1969;44:163-70

11. Klein A. The karyotypesof Ardea cinerea (L.) and Ardea purpurea (L.) and their hybrids. Chrom Inf Serv. 1978;15:14-5.

12. Liu L, Wang B, Guo X, Li F. Chromosomal identification of sex and karyotype analysis in Nipponia nippon. J Beijing Norm Univ (Nat Sci) 1992:28:211-4
13. Misra M, Srivastava MDL Somatic chromosome of Bubulcus lbis (L) (Cattle-egret): a case of reciprocal trans1 ocation. Genetica. 1976;46:155-60.

14. Mohanty MK, Bhunya SP. Karyological studies in 4 species of Ardeid birds (Ardeidae, Ciconiiformes). Genetica. 1990;81:211-4.

15. Sasaki M, Takagi N. Karyotypes of 4 rare species of birds. Chrom Inf Serv. 1974;16:31

16. Takagi N, Sasaki M. A phylogenetic study of bird karyotypes. Chromosoma. 1974:46:91-120.

17. Zhu S, Jiang D. Preliminary karyotype analysis of the birds Ardeidae. Zool Res. 1984:5:79-81.

18. Ellegren $\mathrm{H}$. Evolutionary stasis: the stable chromosomes of birds. Trends Ecol Evol. 2010:25:283-91.

19. Griffin DK, Robertson LBW, Tempest HG, Skinner BM. The evolution of the avian genome as revealed by comparative molecular cytogenetics. Cytogenet Genome Res. 2007;117:64-77.

20. Degrandi TM, Barcellos SA, Costa AL, Garnero ADV, Hass I, Gunski RJ. Introducing the bird chromosome database: an overview of cytogenetic studies in birds. Cytogenet Genome Res. 2020;160:199-205.

21. Zhang G, Li C, Li Q, Li B, Larkin DM, Lee C, Storz JF, Antunes A, Greenwold MJ. Comparative genomics reveals insights into avian genome evolution and adaptation. Science. 2014;346:1311-20.

22. Kretschmer R, Ferguson-Smith MA, de Oliveira EHC. Karyotype evolution in birds: from conventional staining to chromosome painting. Genes. 2018;9:181.

23. Seligmann IC, Furo IO, dos Santos MS, Tagliarini MM, Araujo CCD, O'Brien PCM, Ferguson-Smith MA, de Oliveira EHC. Comparative chromosome painting in two Brazilian stork species with different diploid numbers. Cytogenet Genome Res. 2019;159:32-8.

24. Nie W, O'Brien PCM, Ng BL, Fu B, Volobouev V, Carter NP, Ferguson-Smith MA, Yang F. Avian comparative genomics: Reciprocal chromosome painting between domestic chicken (Gallus gallus) and the stone curlew (Burhinus oedicnemus, Charadriiformes) - An atypical species with low diploid number. Chromosome Res. 2009;17:99-113.

25. Hansmann T, Nanda I, Volobouev V, Yang F, Schartl M, Haaf T, Schmid M. Cross-species chromosome painting corroborates microchromosome fusion during karyotype evolution of birds. Cytogenet Genome Res. 2009;126:281-304.

26. Nie W, O'Brien PCM, Fu B, Wang J, Su W, He K, BedHom B, Volobouev V, Ferguson-Smith MA, Dobigny $G$, et al. Multidirectional chromosome painting substantiates the occurrence of extensive genomic reshuffling within Accipitriformes. BMC Evol Biol. 2015;15:205.

27. Pinheiro MLS, Nagamachi CY, Ribas TFA, Diniz CG, O'Brien PCM, FergusonSmith MA, Yang F, Pieczarka JC. Chromosomal painting of the sandpiper (Actitis macularius) detects several fissions for the Scolopacidae family (Charadriiformes). BMC Ecol Evol. 2021;21:8.

28. Ribas TFA, Pieczarka JC, Griffin DK, Kiazim LG, Nagamachi CY, O’Brien PCM, Ferguson-Smith MA, Yang F, Aleixo A, O'Connor RE. Analysis of multiple chromosomal rearrangements in the genome of Willisornis vidua using BAC-FISH and chromosome painting on a supposed conserved karyotype. BMC Ecol Evol. 2021;21:34

29. van Tuinen M, Hedges SB. Calibration of avian molecular clocks. Mol Biol Evol. 2001;18:206-13.

30. Hackett SJ, Kimball RT, Reddy S, Bowie RCK, Braun EL, Braun MJ, Chojnowski JL, CoxWA, Han K, Harshman J, et al. A phylogenomic study of birds reveals their evolutionary history. Science. 2008;320:1763-7.

31. Jarvis ED, Mirarab S, Aberer AJ, Li B, Houde P, Li C, Ho SYW, Faircloth BC, Nabholz B, Howard JT, et al. Whole-genome analyses resolve early branches in the tree of life of modern birds. Science. 2014;346:1320-31.

32. Wang J, Su R, Su W, Cheng C, Wei S, Ding H, Duan Y, Li S, Nie W. Establishment of cell lines derived from skin biopsies of crested ibis (Nipponia nippon) and their biological characteristics. Zool Res. 2012;33:591-6.

33. Damas J, O'Connor R, Farré M, Lenis V, Martell H, Mandawala A, Fowler K, Joseph S, Swain MT, Griffin DK, et al. Upgrading short-read anima genome assemblies to chromosome level using comparative genomics and a universal probe set. Genome Res. 2017;27:875-84.

34. Furo IO, Kretschmer R, O'Brien PCM, Pereira JCDC, Gunski RJ, Garnero ADV O'Connor RE, Griffin DK, Ferguson-Smith MA, De Oliveira EHC. Cytotaxonomy of Gallinula melanops (Gruiformes, Rallidae): karyotype evolution and phylogenetic inference. Genet Mol Biol. 2021;44(2):e20200241.

35. Furo 10 , Kretschmer R, O'Brien PCM, Pereira J, Garnero ADV, Gunski RJ, O'Connor RE, Griffin DK, Gomes AJB, Ferguson-Smith MA, et al. 
Chromosomal evolution in the phylogenetic context in Neotropical Psittacidae with emphasis on a species with high karyotypic reorganization (Myiopsitta monachus). Front Genet. 2020;11:721.

36. Joseph S, O'Connor RE. Chromosome level genome assembly and comparative genomics between three falcon species reveals an unusual pattern of genome organisation. Diversity. 2018;10:113.

37. Kretschmer R, de Souza MS, Furo IO, Romanov MN, Gunski RJ, Garnero ADV, de Freitas TRO, de Oliveira EHC, O'Connor RE, Griffin DK. Interspecies chromosome mapping in Caprimulgiformes, Piciformes, Suliformes, and Trogoniformes (Aves): cytogenomic insight into microchromosome organization and karyotype evolution in birds. Cells. 2021;10:826.

38. Kretschmer R, Furo IO, Gomes AJB, Kiazim LG, Gunski RJ, Garnero ADV, Pereira JC, Ferguson-Smith MA, de Oliveira HC, Griffin DK, et al. A comprehensive cytogenetic analysis of several members of the family Columbidae (Aves, Columbiformes). Genes. 2020;11:632.

39. Kretschmer R, Gunski RJ, Garnero ADV, de Freitas TRO, Toma GA, Cioffifi MB, de Oliveira EHC, O'Connor RE, Griffin DK. Chromosomal analysis in Crotophaga ani (Aves, Cuculiformes) reveals extensive genomic reorganization and an unusual Z-autosome Robertsonian translocation. Cells. 2021:10:4.

40. O'Connor RE, Farré M, Joseph S, Damas J, Kiazim L, Jennings R, Bennett S, Slack EA, Allanson E, Larkin DM, et al. Chromosome-level assembly reveals extensive rearrangement in saker falcon and budgerigar, but not ostrich, genomes. Genome Biol. 2018;19:171.

41. O'Connor RE, Kiazim L, Skinner B, Fonseka G, Joseph S, Jennings R, Larkin DM, Griffin DK. Patterns of microchromosome organization remain highly conserved throughout avian evolution. Chromosoma. 2019;128:21-9.

42. Kretschmer R, Gunski RJ, Garnero ADV, O'Brien PCM, Ferguson-Smith MA, de Freitas TRO, De Oliveira EHC. Chromosome painting in Vanellus chilensis: Detection of a fusion common to clade Charadrii (Charadriiformes). Cytogenet Genome Res. 2015;146:58-63.

43. Kretschmer R, de Souza MS, Barcellos SA, Degrandi TM, Pereira J, O'Brien PCM, Ferguson-Smith MA, Gunski RJ, Garnero ADV, de Oliveira EHC, et al. Novel insights into chromosome evolution of Charadriiformes: extensive genomic reshuffling in the wattled jacana (Jacana jacana, Charadriiformes, Jacanidae). Genet Mol Biol. 2020;43(1):e20190236.

44. Ericson PGP, Anderson CL, Britton T, Elzanowski A, Johansson US, Källersjö M, Ohlson JI, Parsons TJ, Zuccon D, Mayr G. Diversification of Neoaves: integration of molecular sequence data and fossils. Biol Lett. 2006; 2:543-7.

45. Burleigh JG, Kimball RT, Braun EL. Building the avian tree of life using a large-scale, sparse supermatrix. Mol Phylogenet Evol. 2015;84:53-63.

46. Gibb GC, Martyn K, Penny D. Beyond phylogeny: pelecaniform and ciconiiform birds, and long-term niche stability. Mol Phylogenet Evol. 2013;68:229-38

47. Kimball RT, Oliveros $C H$, Wang N, White ND, Barker FK, Field DJ, Ksepka DT, Chesser RT, Moyle RG, Braun MJ, et al. A phylogenomic supertree of birds. Diversity. 2019;11:109.

48. Kuhl H, Frankl-Vilches C, Bakker A, Mayr G, Nikolaus G, Boerno ST, Klages V, Timmermann B, Gahr M. An unbiased molecular approach using 3'UTRs resolves the avian family-level tree of life. Mol Biol Evol. 2021;38:108-27.

49. Prum RO, Berv JS, Dornburg A, Field DJ, Townsend JP, Lemmon EM, Lemmon AR. A comprehensive phylogeny of birds (Aves) using targeted next-generation DNA sequencing. Nature. 2015;526:569-73.

\section{Publisher's Note}

Springer Nature remains neutral with regard to jurisdictional claims in published maps and institutional affiliations.

Ready to submit your research? Choose BMC and benefit from:

- fast, convenient online submission

- thorough peer review by experienced researchers in your field

- rapid publication on acceptance

- support for research data, including large and complex data types

- gold Open Access which fosters wider collaboration and increased citations

- maximum visibility for your research: over $100 \mathrm{M}$ website views per year

At BMC, research is always in progress.

Learn more biomedcentral.com/submissions 